
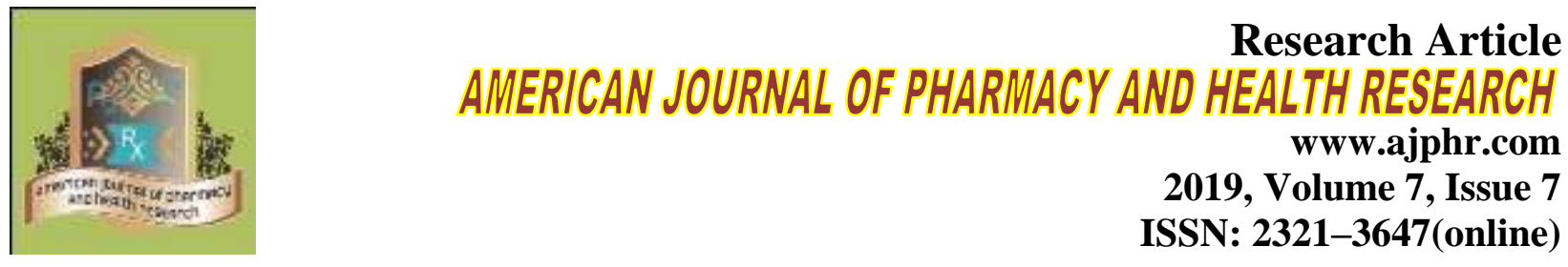

\title{
Synthesis, Characterization of substituted 4-(bromomethyl)-N-(4- ethyl-6-methylpyrimidin-2-yl)benzamide Derivatives and Evaluation of their Anti-microbial activity.
}

\author{
M.Vijaya Laxmi ${ }^{1}$, Guguloth Ravi ${ }^{2 *}$, A.Ravinder Nath ${ }^{1 *}$ \\ 1.Department of Pharmacy \& Bio-Technology, University College of Technology, Osmania \\ University, Hyderabad-500007, Telanagana State. \\ 2.Department of Chemistry, University College of Science, Osmania University, Hyderabad- \\ 500007, Telangana State
}

\begin{abstract}
The substituted 4-(bromomethyl)-N-(4-ethyl-6-methylpyrimidin-2-yl) benzamide derivatives 6a$\mathbf{k}$ has been synthesis from reaction in between substituted 4-ethyl-6-methylpyrimidin-2-amine and 4-(bromomethyl)benzoyl bromide 5 in presence of $\mathrm{KOH}$. Type of aldol condensation reaction in between aldehydes and ketones to form $\alpha, \beta$-Unsaturated carbonyl compounds 3 this compound has been reacted with guanidine in presence of dry alcohol to convert 4-ethyl-6methylpyrimidin-2-amine4.Chemical structures of all the new compounds were established by IR, 1H, 13C NMR, MS and elemental data. The compounds 6a-k were evaluated for their antibacterial activity against Gram-positive bacteria viz. Bacillus subtilis, Bacillus sphaericus and Staphylococcus aureus, and three Gram-negative bacteria viz. Pseudomonas aeruginosa, Klebsiella aerogenes and Chromobacterium violaceum and also evaluate their antifungal activity against Candida albicans (C.albicans)(ATCC 10231), Aspergillus fumigates(A.fumigatus) (HIC 6094), Trichophyton rubrum(T. rubrum) (IFO 9185), and Trichophyton mentagrophytes(T. mentagrophytes) (IFO 40996) Amongst them, compounds containing [3-hydrophenyl] moiety 6d, [3-chlorophenyl] moiety 6f and [4-nitrophenyl] moiety 6h showed significant antibacterial and antifungal activity, almost equal/more than the activity of the standard drugs Streptomycin and Amphotericin-B. Further, the compounds 6a-k were also screened for Most of these new compounds showed appreciable activity against test bacteria and fungi and emerged as potential molecules for further development.
\end{abstract}

Keywords: Synthesis,4-(bromomethyl)-N-(4-ethyl-6-methylpyrimidin-2-yl) Benzamide, antibacterial activity, antifungal activity.

*Corresponding Author Email: drraviguguloth@gmail.com

Received 04 July 2019, Accepted 13 July 2019

Please cite this article as: G Ravi et al., Synthesis, Characterization of substituted 4-(bromomethyl)-N(4-ethyl-6-methylpyrimidin-2-yl)benzamide Derivatives and Evaluation of their Anti-microbial activity . American Journal of Pharmacy \& Health Research 2019. 


\section{INTRODUCTION}

Heterocyclic compounds corresponding to one of the most active classes of compounds possessing a wide range of biological activities, including antibacterial, antifungal, and other biological activities[1-10] Further, the treatment of infectious diseases still remains an important and challenging problem because of a combination of factors including emerging infectious diseases and the increasing number of multi-drug resistant microbial pathogens. In spite of a large number of antibiotics and chemotherapeutics available for medical use, at the same time the emergence of old and new antibiotic resistance created in the last decades revealed a substantial medical need for new classes of antimicrobial agents. There is a real perceived need for the discovery of new compounds endowed with antimicrobial activity. Similarly in recent decades, an increased incidence of fungal infections has been observed as a consequence of the growing number of immune compromised patients and the frequent use of antibacterial and cytotoxic drugs. For many fungal infections, polyenes, such as amphotericin B, represent the standard therapy. Mycobacterium tuberculosis[11]. Benzamie derivatives are also known to exhibit diverse bioactivities such as anti-convulsant[12], antidiarrheal[13], antihistaminic[14], anti-diabetic[15], cyclooxy genase (COX) inhibitory[16], Ca2+-channel blocker[17], cardioprotective[18], anti-ischemic[19], anti-cancer[20].The synthesis of heterocycles containing multi structure in a molecule has received much attention in recent years[21]. However, literature survey revealed that linked heterocycles containing Benzamide have been reported. Based on the wide range spectrum of biological profile of Benzamide and their increasing importance in pharmaceutical, and biological field, and in continuation of our on going research on biologically active heterocycles[22-24] it was thought of interest to accommodate benzamide moieties in a single molecular frame work to synthesize some new heterocyclic compounds with potential biological activity.

The present investigation deals with the synthesis of some new 4-(bromomethyl)-N-(4-ethyl-6methylpyrimidin-2-yl) benzamide derivatives 6a-k in good yields, from substituted 4-ethyl-6methylpyrimidin-2-amine 5 and 4-(bromomethyl)benzoyl bromide 4.The antibacterial and antifungal activities of the compounds 6a-k have also been evaluated.

\section{RESULTS AND DISCUSSION}

The present investigation deals with the synthesis of some new preparation of substituted 4(bromomethyl)-N-(4-ethyl-6-methylpyrimidin-2-yl)benzamide(I-6a-k) in (60-78\%) good yields, 
from starting material carbonyl compounds (I-1\&2). The antibacterial and antifungal activities of the compounds I-6(a-k) have also been evaluated.

Synthesis of the $\alpha, \beta$-Unsaturated carbonyl compounds(I-3) commenced from condensation in presence of basic condition $(\mathrm{NaOH})$ under reflux condition at $60-80^{\circ} \mathrm{C}$ for $3-4 \mathrm{hrs}$ in between ketone and substituted aldehydes(I-1\&2)(Scheme-1). The key intermediate has been prepared in excellent yields $(86 \%)$. The cyclization of compound 3 with Guanidine in presence of dry alcohol at reflux for 4-6 hrs, furnished the 4-ethyl-6-methylpyrimidin-2-amine in 84\%, yield (Scheme-2) M.P: $128-130^{\circ} \mathrm{C}$. The IR spectrum of compound 4 showed absorption bands at $3468,3445(\mathrm{~d}) \mathrm{cm}^{-}$ 1 assignable to primary amine functional group $\left(\mathrm{NH}_{2}\right)$, The IR spectrum of compound 27 showed absorption bands $3350(\mathrm{~s}) \mathrm{cm}^{-1}$ assignable $2^{0}$-amine provide a strong evidence that $1^{0}$-amine undergoes to cyclization reaction.

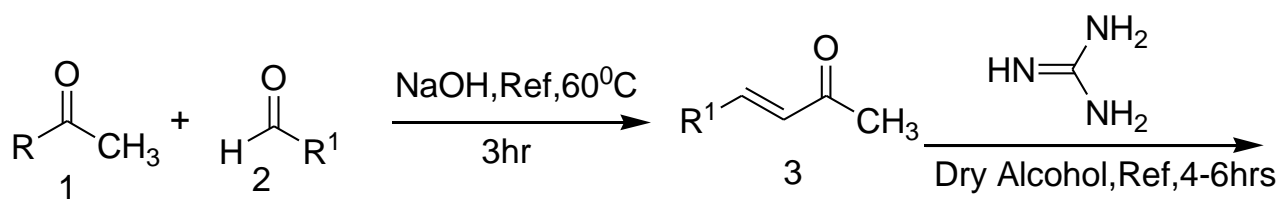<smiles>[R]c1cc([R])nc(N)n1</smiles><smiles>[R]c1cc([R])nc(NC(=O)c2ccc(CBr)cc2)n1</smiles>

Scheme 1. Synthetic pathways for compounds 6a-k

\section{Compound 6:}<smiles>[R]c1cc([R])nc(NC(=O)c2ccc(CBr)cc2)n1</smiles>

Table 1:

\begin{tabular}{lllllll}
\hline $\begin{array}{l}\text { Compound } \\
\text { No }\end{array}$ & $\mathbf{R}$ & $\mathbf{R}^{\mathbf{1}}$ & $\begin{array}{l}\text { Molecular } \\
\text { Formula }\end{array}$ & $\begin{array}{l}\text { Molecular } \\
\text { Weight }\end{array}$ & Yield(\%) & M.P( $\left.{ }^{\boldsymbol{0}} \mathbf{C}\right)$ \\
\hline $6 \mathrm{a}$ & $\mathrm{CH}_{3}$ & $\mathrm{C}_{2} \mathrm{H}_{5}$ & $\mathrm{C}_{15} \mathrm{H}_{16} \mathrm{~N}_{3} \mathrm{OBr}$ & 333 & 78 & $144-146$ \\
$6 \mathrm{~b}$ & $\mathrm{CH}_{3}$ & $\mathrm{C}_{3} \mathrm{H}_{7}$ & $\mathrm{C}_{16} \mathrm{H}_{18} \mathrm{~N}_{3} \mathrm{OBr}$ & 347 & 76 & $158-160$ \\
$6 \mathrm{c}$ & $\mathrm{CH}_{3}$ & $\mathrm{C}_{6} \mathrm{H}_{5}$ & $\mathrm{C}_{19} \mathrm{H}_{16} \mathrm{~N}_{3} \mathrm{OBr}$ & 381 & 68 & $152-154$ \\
6d & $\mathrm{CH}_{3}$ & $3-\mathrm{OH}-\mathrm{Ph}$ & $\mathrm{C}_{19} \mathrm{H}_{16} \mathrm{~N}_{3} \mathrm{O}_{2} \mathrm{Br}$ & 397 & 64 & $160-162$ \\
\hline
\end{tabular}




\begin{tabular}{lllllll}
\hline $6 \mathrm{e}$ & $\mathrm{CH}_{3}$ & $4-\mathrm{OH}-\mathrm{Ph}$ & $\mathrm{C}_{19} \mathrm{H}_{16} \mathrm{~N}_{3} \mathrm{O}_{2} \mathrm{Br}$ & 397 & 65 & $172-174$ \\
$6 \mathrm{f}$ & $\mathrm{CH}_{3}$ & $3-\mathrm{Cl}-\mathrm{Ph}$ & $\mathrm{C}_{19} \mathrm{H}_{15} \mathrm{~N}_{3} \mathrm{OClBr}$ & 413.5 & 74 & $174-176$ \\
$6 \mathrm{~g}$ & $\mathrm{CH}_{3}$ & $4-\mathrm{Cl}-\mathrm{Ph}$ & $\mathrm{C}_{19} \mathrm{H}_{15} \mathrm{~N}_{3} \mathrm{OClBr}$ & 413.5 & 72 & $172-174$ \\
$6 \mathrm{~h}$ & $\mathrm{CH}_{3}$ & $4-\mathrm{NO}_{2}-\mathrm{Ph}$ & $\mathrm{C}_{19} \mathrm{H}_{15} \mathrm{~N}_{4} \mathrm{O}_{3} \mathrm{Br}$ & 426 & 68 & $162-164$ \\
$6 \mathrm{i}$ & $\mathrm{CH}_{3}$ & $4-\mathrm{CH}_{3}-\mathrm{Ph}$ & $\mathrm{C}_{20} \mathrm{H}_{18} \mathrm{~N}_{3} \mathrm{OBr}$ & 395 & 62 & $170-172$ \\
$6 \mathrm{j}$ & $\mathrm{CH}_{3}$ & $4-\mathrm{F}-\mathrm{Ph}$ & $\mathrm{C}_{19} \mathrm{H}_{15} \mathrm{~N}_{3} \mathrm{OBrF}$ & 398 & 64 & $168-170$ \\
$6 \mathrm{k}$ & $\mathrm{CH}_{3}$ & $4-\mathrm{Br}-\mathrm{Ph}$ & $\mathrm{C}_{19} \mathrm{H}_{15} \mathrm{~N}_{3} \mathrm{OBr}$ & 459 & 60 & $184-186$ \\
\hline
\end{tabular}

\section{Antimicrobial Activity:}

\section{Antibacterial Activity:}

The in vitro antibacterial activity 4-(bromomethyl)-N-(4-ethyl-6-methylpyrimidin-2yl)benzamide 6(a-k) was assessed against three representative Gram-positive bacteria viz. Bacillus subtilis, Bacillus sphaericus and Staphylococcus aureus, and three Gram-negative bacteria viz. Pseudomonas aeruginosa, Klebsiella aerogenes and Chromobacterium violaceum by the disc diffusion method in DMSO[25].In the series of $\mathbf{6}(\mathbf{a}-\mathbf{k})$, the compounds $\mathbf{6 d , 6 f}$ and $\mathbf{6 h}$ are found to be the most active against Gram-positive bacteria and the Gram-negative bacteria [Table 1]. The remaining compounds showed moderate to good activity against all the Grampositive bacteria and the Gram-negative bacteria.

Table 1: Antibacterial activity of compounds 6(a-k).

\begin{tabular}{|c|c|c|c|c|c|c|}
\hline \multirow[t]{2}{*}{ Compd. } & \multicolumn{6}{|c|}{ Minimum Inhibitory Concentration (MIC) in $\mu \mathrm{g} / \mathrm{mL}$} \\
\hline & $\begin{array}{l}\text { B. } \\
\text { subtilis }\end{array}$ & $\begin{array}{l}\text { B. } \\
\text { sphaericus }\end{array}$ & $\begin{array}{l}\text { S. } \\
\text { aureus }\end{array}$ & $\begin{array}{l}P . \\
\text { aeruginosa }\end{array}$ & $\begin{array}{l}\text { K. } \\
\text { aerogenes }\end{array}$ & $\begin{array}{l}\text { C. } \\
\text { violaceum }\end{array}$ \\
\hline $6 a$ & 24 & 26 & 26 & 22 & 26 & 22 \\
\hline $6 b$ & 20 & 22 & 22 & 24 & 22 & 20 \\
\hline $6 c$ & 24 & 26 & 22 & 22 & 24 & 26 \\
\hline $6 \mathrm{~d}$ & 32 & 28 & 30 & 28 & 32 & 30 \\
\hline $6 e$ & 30 & 32 & 28 & 30 & 28 & 26 \\
\hline $6 f$ & 32 & 30 & 30 & 32 & 30 & 32 \\
\hline $6 g$ & 30 & 28 & 28 & 30 & 28 & 28 \\
\hline $6 \mathrm{~h}$ & 32 & 28 & 30 & 32 & 28 & 30 \\
\hline $6 \mathrm{i}$ & 24 & 26 & 22 & 24 & 22 & 25 \\
\hline $6 \mathrm{j}$ & 30 & 28 & 30 & 28 & 30 & 28 \\
\hline $6 \mathrm{k}$ & 30 & 28 & 30 & 28 & 30 & 28 \\
\hline SM & 32 & 30 & 28 & 30 & 32 & 28 \\
\hline $\mathrm{SM}=$ & ptomycir & & & & & \\
\hline
\end{tabular}

Graphical Data of Anti-Bacterial Activity: 


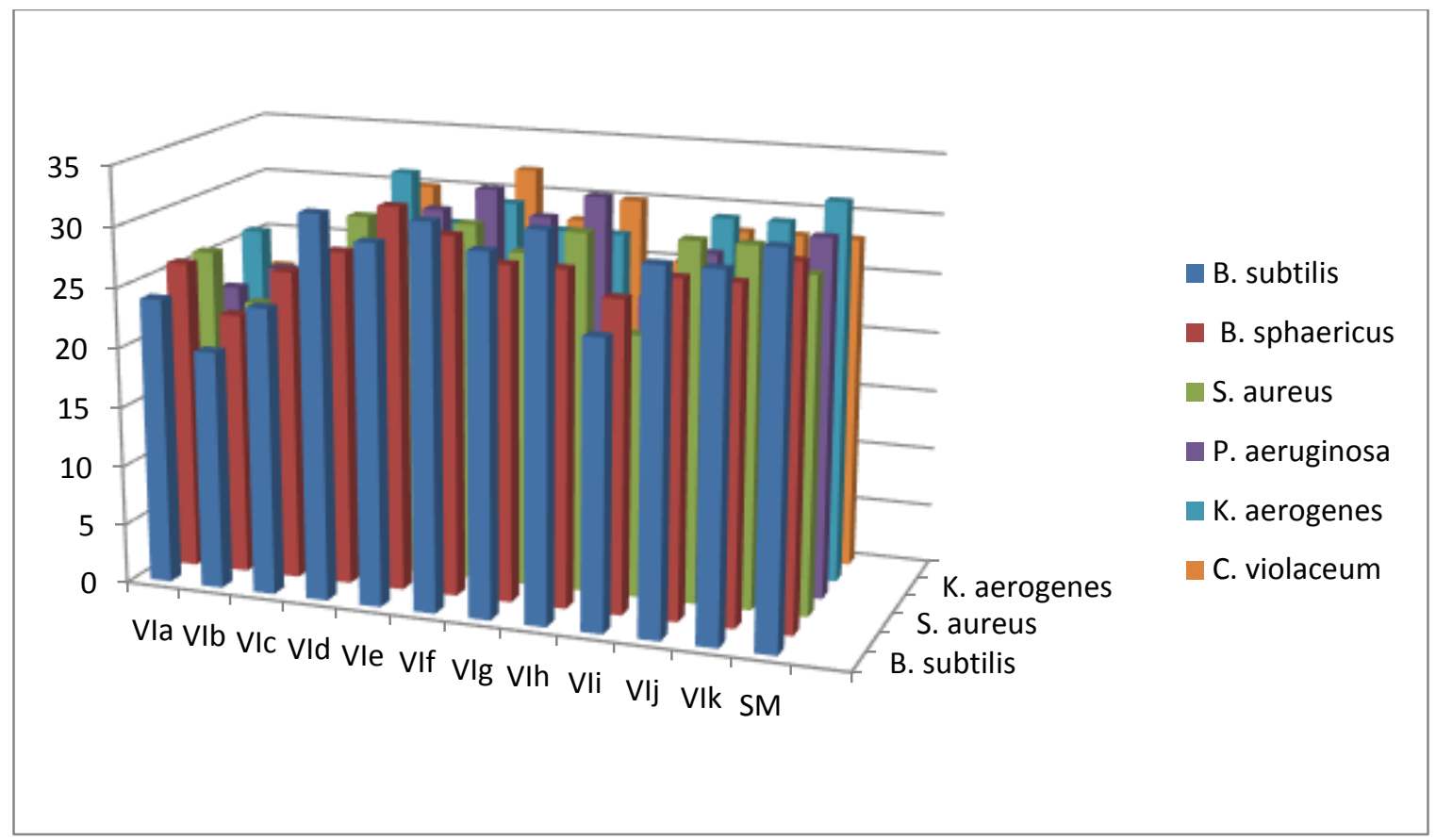

\section{Antifungal Activity}

The compounds $\mathbf{6}(\mathbf{a}-\mathbf{k})$ were also screened for their antifungal activity against Candida albicans (C.albicans)(ATCC 10231), Aspergillus fumigates(A.fumigatus) (HIC 6094), Trichophyton rubrum(T. rubrum) (IFO 9185), and Trichophyton mentagrophytes(T. mentagrophytes) (IFO 40996) in dimethyl sulfoxide (DMSO) by disc difusion method. Amphotericin B was used as a standard drug. The antifungal screening data showed appreciable activity of the test compounds. Among the screened compounds $\mathbf{6 d , 6 f}$ and $\mathbf{6 h}$ showed good antifungal activity[Table 2].Remaining compounds showed moderate anti fungal activity against test compounds.

Table 2. Antifungal Activity of Compounds 6(a-k)

\begin{tabular}{lllll}
\hline Compound & \multicolumn{4}{c}{ Mean zone inhibition (MZI) in $\mathbf{1 0} \boldsymbol{\mu g}$ / $\mathbf{m L}$} \\
& C.albicans & A. fumigatus & T. rubrum & T. mentagropytes \\
\hline 6a & 22 & 18 & 18 & 20 \\
6b & 20 & 18 & 16 & 20 \\
6c & 22 & 24 & 26 & 24 \\
6d & 28 & 30 & 28 & 28 \\
6e & 28 & 28 & 26 & 26 \\
6f & 30 & 28 & 26 & 28 \\
6g & 28 & 28 & 26 & 26 \\
6h & 26 & 30 & 26 & 28 \\
6i & 18 & 18 & 22 & 21 \\
6j & 28 & 28 & 24 & 28 \\
6k & 30 & 26 & 26 & 28 \\
Amphotericin & 28 & 30 & 26 & 28 \\
B & & & & \\
\hline
\end{tabular}

${ }^{\mathrm{a}}$ Values are mean $(\mathrm{n}=3)$. 


\section{Graphical data of Anti-Fungal activity:}

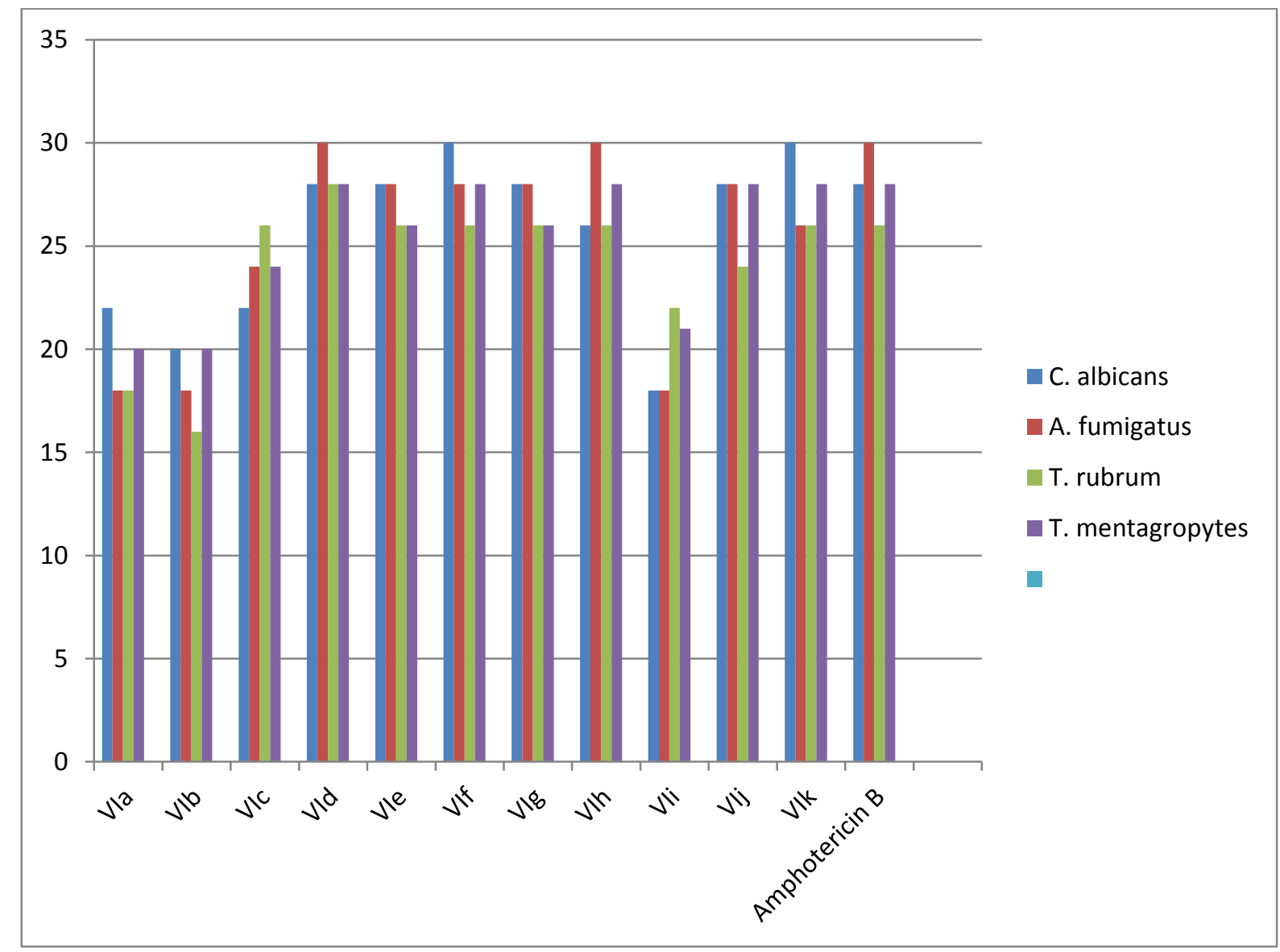

\section{CONCLUSION}

In conclusion, a series of activity 4-(bromomethyl)-N-(4-ethyl-6-methylpyrimidin-2yl)Benzamide derivatives $\mathbf{6}(\mathbf{a}-\mathbf{k})$ were prepared. The antibacterial activity of these compounds was evaluated against various bacteria. The compounds showed variable degree of antimicrobial activity. Among the screened compounds $6 \mathbf{d}, \mathbf{6} \mathbf{f}$ and $\mathbf{6 h}$ were found to be the most active or almost equal active against all the microorganisms employed both for antibacterial and antifungal activity. Further, these compounds showed appreciable activity against the test fungi, and emerged as potential molecules for further development.

\section{EXPERIMENTAL:}

In this experiment all reagents are used analytical reagent grade obtained from Sigma-Aldrich, Merck, SD fine and avira chemicals. With using standard procedures we purified Water, methanol, acetone, ether etc 4-(bromomethyl)-N-(4-ethyl-6-methylpyrimidin-2-yl)Benzamide derivatives. ${ }^{1} \mathrm{H}$ NMR and ${ }^{13} \mathrm{C}$ NMR spectra were recorded on Bruker $400 \mathrm{MHz}$ NMR instrument using tetra methyl silane (TMS) as internal standard compound and coupling constants $(J)$ are reported in Hz units.VG AUTOSPEC mass spectrometer. Electronic spectra of all compounds 
were recorded on Schimadzu UV-Vis 1601 spectrophotometer. ESI mass spectra were Melting points of the ligands and metal complexes decomposition temperature were determined on Polmon instrument (Model No. MP-102). IR spectra of the compounds were recorded using $\mathrm{KBr}$ pellets in the range $4000-600 \mathrm{~cm}^{1}$ on Perkin-Elmer Infrared model 337. The percentage composition of $\mathrm{C}, \mathrm{H}, \mathrm{N}$ of the compounds were determined by using micro analytical techniques on Perkin Elmer 240C (USA) elemental analyzer. All reactions were monitored by thin-layer chromatography (TLC) on pre-coated silica gel F254 plates from Merck, and compounds visualized either by exposure to UV light. Chromatographic columns 60-120 mesh silica gel for separations were used. Elemental analyses $(\mathrm{C}, \mathrm{H}, \mathrm{N})$ determined by means of a Perkin-Elmer $240 \mathrm{C}, \mathrm{H}, \mathrm{N}$ and $\mathrm{O}$ elemental analyzer, were within $\pm 0.4 \%$ of Perkin-Elmer theory.

General procedure for the preparation of 4-ethyl-6-methylpyrimidin-2-amine (4):

To a vigorously stirring solution of $\alpha, \beta$-unsaturated carbonyl compound; 0.01 mole) in $\mathrm{NaOH}(20 \mathrm{ml}, 20 \mathrm{~N})$ solution and guanidine $(0.01 \mathrm{~mol})$ in presence of dry alcohol were added to reaction mixture. After complete addition, the solution was stirred for another $30 \mathrm{~min}$ at $\mathrm{RT}\left(35^{\circ} \mathrm{C}\right)$. Now this reaction reflux at $60-80^{\circ} \mathrm{C}$ for $4-6 \mathrm{hrs}$. The resulting mixture was poured onto crushed ice $(100 \mathrm{ml})$ with stirring. The product was extracted with chloroform $(4 \mathrm{x} 20 \mathrm{ml})$, which on evaporation yielded a yellow product. This crude product was used for the next step with purification. Recrystallization with ethyl alcohol.

IR (KBr) $\left(\mathbf{c m}^{-1}\right) v_{\max }: 3468,3445,3123,2998,2700,2596,1450,1260,875 .{ }^{\mathbf{1}} \mathbf{H}$ NMR (DMSO-d6, 300 $\mathrm{MHz}) \boldsymbol{\delta}: 1.24\left(\mathrm{t}, 3 \mathrm{H},-\mathrm{CH}_{3}\right), 2.35\left(\mathrm{t}, 3 \mathrm{H}, \mathrm{CH}_{3}\right), 2.80\left(\mathrm{q}, 2 \mathrm{H},-\mathrm{CH}_{2}\right), 4.1\left(\mathrm{~s}, 2 \mathrm{H}, \mathrm{NH}_{2}\right), 6.58(\mathrm{~s}, J=7.8 \mathrm{~Hz}$, 1H,Ar-H,). ${ }^{13} \mathbf{C}$ NMR (DMSO-d6,75 MHz)ס:14.6,29.3,101.9,162.2,167.1,167.9.Mass

(CHCl3) $\lambda_{\max /} \mathrm{nm}\left(\mathrm{cm}^{-1}\right): \mathrm{m} / z, 137\left(\mathrm{M}^{+}\right)$.Anal. Calcd. for $\mathrm{C}_{7} \mathrm{H}_{11} \mathrm{~N}_{3} \mathrm{C}, 78.13 ; \mathrm{H}, 10.11 ; \mathrm{N}, 18.14$. Found: C, 75.15; H,9.37; N, 16.90. Yield: $84 \%$, M.P: $128-130^{\circ} \mathrm{C}$.

General procedure for the preparation of Substituted 4-(bromomethyl)-N-(4-ethyl-6methylpyrimidin-2-yl)benzamide derivatives $(6 a-k)$.

4-ethyl-6-methylpyrimidin-2-amine (4;0.01 mole) was dissolved in $10 \mathrm{ml}$ of potassium hydroxide solution $(0.01 \mathrm{~mol})$ and add dry alcohol slowly in a drop-wise manner. Reflux at 60$80^{\circ} \mathrm{C}$ for 6-8 hrs. This was poured into crushed ice and extracted with chloroform. The chloroform extract was evaporated to get Substituted 4-(bromomethyl)-N-(4-ethyl-6methylpyrimidin-2-yl)benzamide derivatives(I-6a-k).

4-(bromomethyl)-N-(4-ethyl-6-methylpyrimidin-2-yl)benzamide derivatives(6a)

$\operatorname{IR}(\mathbf{K B r}) \quad\left(\mathbf{c m}^{-1}\right) \quad v_{\max }: 3350.1,3080.2,2980.5,2650.2,2100.6,1758.2,1450.2,1202,958 .{ }^{1} \mathbf{H} \quad$ NMR (DMSO-d6, $300 \mathrm{MHz}) \boldsymbol{\delta}: 1.20\left(\mathrm{t}, 3 \mathrm{H},-\mathrm{CH}_{3}\right), 1.98\left(\mathrm{~s}, 3 \mathrm{H}, \mathrm{CH}_{3}\right), 3.8\left(\mathrm{q}, 2 \mathrm{H},-\mathrm{CH}_{2}\right), 4.6(\mathrm{~s}, 1 \mathrm{H}, \mathrm{NH}), 6.6(\mathrm{~s}, J$ 
$=\quad 7.8 \quad \mathrm{~Hz}, \quad 1 \mathrm{H}, \mathrm{Ar}-\mathrm{H}), 6.8-7.83(\mathrm{~m}, 4 \mathrm{H}, \mathrm{Ar}-\mathrm{H}) .{ }^{13} \mathbf{C} \quad$ NMR $\quad$ (DMSO-d6,75

MHz)ס:14.6,25.1,36.7,41.2,101.9,114.2,127.8,120.2,134.4,141.4,156.9,159.4,167.1,167.9.

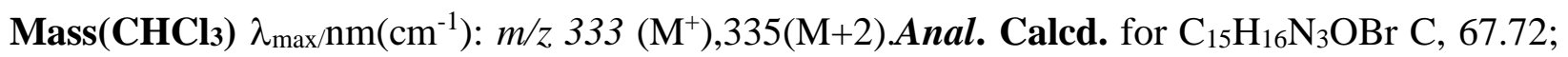
H, 8.32; N, 12.11;O,6.89. Found: C, 65.72; H, 7.42; N, 11.01; O,5.14 Yield: 78\% , M.P: 144$146^{0} \mathrm{C}$.

preparation of 4-(bromomethyl)-N-(4-methyl-6-propylpyrimidin-2-yl)benzamide (6b)

IR(KBr) (cm-1) $v_{\max }: 3330,3075,2970,2640,2080,1708,1440,1190,942 .{ }^{1}$ H NMR (DMSO-d6, 300 $\mathrm{MHz})$

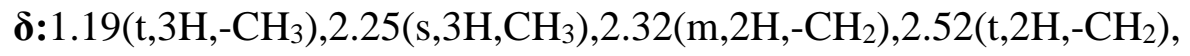
4.26(s,2H,CH 2$), 4.56(\mathrm{~s}, 1 \mathrm{H}, \mathrm{NH}), 6.18(\mathrm{~s}, J=7.8 \mathrm{~Hz}, 1 \mathrm{H}, \mathrm{Ar}-\mathrm{H}), 6.8-7.83(\mathrm{~m}, 4 \mathrm{H}, \mathrm{Ar}-\mathrm{H}) .{ }^{13} \mathbf{C} \quad$ NMR (DMSO-d6,75MHz)反:13.2,22.1,26.5,30.0,33.6,106.9, 125.8,126.2,132.4,136.8,139.4,152.9,

160.8, 163.1,166.9.Mass $\left(\mathbf{C H C l}_{3}\right) \quad \lambda_{\max / \mathrm{nm}\left(\mathrm{cm}^{-1}\right):} \mathrm{m} / z \quad 347 \quad\left(\mathrm{M}^{+}\right), 349(M+2)$.Anal. Calcd. for $\mathrm{C}_{16} \mathrm{H}_{18} \mathrm{~N}_{3} \mathrm{OBr} \mathrm{C}, 68.21 ; \mathrm{H}, 9.16$; N, 10.08.Found: C, 69.83; H, 8.82; N, 08.72. Yield: 76\% , M.P: $158-160^{\circ} \mathrm{C}$.

Preparation of 4-(bromomethyl)-N-(4-methyl-6-phenylpyrimidin-2-yl)benzamide(6c)

IR (KBr) $\left(\mathbf{c m}^{-1}\right) v_{\max }: 3372,3092,2985,2632,2130,1758,1458,1210,736 .{ }^{1} \mathbf{H}$ NMR (DMSO-d6, $300 \mathrm{MHz}) \boldsymbol{\delta}: 1.45\left(\mathrm{~s}, 3 \mathrm{H},-\mathrm{CH}_{3}\right), 3.65\left(\mathrm{~s}, 2 \mathrm{H}, \mathrm{CH}_{2}\right), 4.4(\mathrm{~s}, 1 \mathrm{H}, \mathrm{NH}), 6.5(\mathrm{~s}, J=7.8 \mathrm{~Hz}, 1 \mathrm{H}, \mathrm{Ar}-\mathrm{H}), 6.8-$ 7.8(m,9H,Ar-H). ${ }^{\mathbf{1 3}} \mathbf{C} \quad$ NMR (DMSO-d6,75 MHz) $\boldsymbol{\delta}: 16.6,29.1, \quad 110.2,121.8,122.3,125.3$, 130.2,132.4,134.1,137.8,140.5,141.4,144.3,148.9,152.3,155.9,163.8, 165.1, 166.2.Mass(CHCl $)$ $\lambda_{\max /} \mathrm{nm}\left(\mathrm{cm}^{-1}\right): m / z 381\left(\mathrm{M}^{+}\right), 383(M+2)$.Anal. Calcd. for $\mathrm{C}_{19} \mathrm{H}_{16} \mathrm{~N}_{3} \mathrm{OBr} \mathrm{C}, 70.38 ; \mathrm{H}, 10.15 ; \mathrm{N}$, 13.13. Found: C, 69.12; H,9.42; N, 10.01. Yield: 68\% , M.P: $152-154^{0} \mathrm{C}$.

Preparation of 4-(bromomethyl)-N-(4-(3-hydroxyphenyl)-6-methylpyrimidin-2-yl)benzamide (6d)

$\operatorname{IR}(\mathbf{K B r})\left(\mathbf{c m}^{-1}\right) v_{\max }: 3315,3020,2950,2621,2008,1685,1402,1132,940 .{ }^{1} \mathbf{H}$ NMR (DMSO-d6,300 $\mathrm{MHz}) \quad \delta: 1.18\left(\mathrm{~s}, 3 \mathrm{H},-\mathrm{CH}_{3}\right), 2.20\left(\mathrm{~s}, 2 \mathrm{H}, \mathrm{CH}_{2}\right), 4.36(\mathrm{~s}, 1 \mathrm{H}, \mathrm{NH}), 6.56(\mathrm{~s}, \quad J=7.8 \mathrm{~Hz}, 1 \mathrm{H}, \mathrm{Ar}-\mathrm{H}), 6.8-$ 7.8(m,8H,Ar-H),8.1(s,1H,OH). ${ }^{13} \mathbf{C} \quad$ NMR $\quad$ (DMSO-d6,75 $\quad$ MHz) $\quad \boldsymbol{\delta}: 12.6,22.1,112.9$, 122.8,128.8,130.4,137.8,138.8,140.2,141.4,145.8,146.9,150.4,154.3,158.9,160.8,163.1,164.9,170 .4.Mass $\left(\mathbf{C H C l}_{3}\right) \quad \lambda_{\max /} / \mathrm{nm}\left(\mathrm{cm}^{-1}\right): \quad m / z \quad 397\left(\mathrm{M}^{+}\right), 399(M+2)$.Anal. Calcd. for $\mathrm{C}_{19} \mathrm{H}_{16} \mathrm{~N}_{3} \mathrm{O}_{2} \mathrm{Br}$ C,70.18;H,11.52;N,10.12. Found:C,69.42;H,8.02;N,10.01, Yield: 64\% , M.P: $160-162^{0}$ C.

Preparation of 4-(bromomethyl)-N-(4-(4-hydroxyphenyl)-6-methylpyrimidin-2-yl)benzamide (6e)

IR (KBr) (cm-1) $v_{\max }: 3320,3010,2960,2631,2098,1635,1412,1132,940 .{ }^{\mathbf{1}} \mathbf{H}$ NMR (DMSO-d6,300 $\mathrm{MHz}) \quad \delta: 1.20\left(\mathrm{~s}, 3 \mathrm{H},-\mathrm{CH}_{3}\right), 2.25\left(\mathrm{~s}, 2 \mathrm{H}, \mathrm{CH}_{2}\right), 4.40(\mathrm{~s}, 1 \mathrm{H}, \mathrm{NH}), 6.36(\mathrm{~s}, \quad J=7.8 \mathrm{~Hz}, 1 \mathrm{H}, \mathrm{Ar}-\mathrm{H}), 6.8-$ 
7.8(m,8H,Ar-H),8.1(s,1H,OH). ${ }^{\mathbf{1 3}} \mathbf{C} \quad$ NMR $\quad$ (DMSO-d $6,75 \quad$ MHz) $\quad \boldsymbol{\delta}: 12.6,22.1,112.9$, 122.8,128.8,130.4,137.8,138.8,140.2,141.4,145.8,146.9,150.4,154.3,158.9,160.8,163.1,164.9,170 .4.Mass $\left(\mathbf{C H C l}_{3}\right) \lambda_{\max / n m}\left(\mathrm{~cm}^{-1}\right): m / z, 397\left(\mathrm{M}^{+}\right), 399(M+2)$.Anal. Calcd. for $\mathrm{C}_{19} \mathrm{H}_{16} \mathrm{~N}_{3} \mathrm{O}_{2} \mathrm{Br} \mathrm{C}$, 69.72; H,11.32; N,09.11. Found: C, 68.83; H,10.97; N, 10.01. Yield: 65\% , M.P: $172-174^{0}$ C.

Preparation of 4-(bromomethyl)-N-(4-(3-chlorophenyl)-6-methylpyrimidin-2-yl)benzamide (6f).

IR (KBr)(cm $\left.{ }^{-1}\right) v_{\max }: 3421,3105,2982,2612,2120,1762,1320,1210,940 .{ }^{\mathbf{1}} \mathbf{H}$ NMR (DMSO-d6, $300 \mathrm{MHz}) \boldsymbol{\delta}: 1.10\left(\mathrm{~s}, 3 \mathrm{H},-\mathrm{CH}_{3}\right), 2.40\left(\mathrm{~s}, 3 \mathrm{H}, \mathrm{CH}_{3}\right), 4.30(\mathrm{~s}, 1 \mathrm{H}, \mathrm{NH}), 6.28(\mathrm{~s}, J=7.8 \mathrm{~Hz}, 1 \mathrm{H}, \mathrm{Ar}-\mathrm{H}), 6.8-$ 7.82(m,10H,Ar-H) ${ }^{13} \mathbf{C} \quad$ NMR (DMSO-d6,75 MHz) $\quad \boldsymbol{\delta}: 16.6,28.1,112.9,120.8$, 130.2,131.4,134.4,136.9,140.4,142.6,144.8,151.8,154.6,155.8,156.6,162.8, 164.6,168.0.Mass(C $\left.\mathrm{HCl}_{3}\right) \lambda_{\max } / \mathrm{nm}\left(\mathrm{cm}^{-1}\right): \mathrm{m} / z 413.5\left(\mathrm{M}^{+}\right), 415.5(M+2)$.Anal. Calcd. for $\mathrm{C}_{19} \mathrm{H}_{15} \mathrm{~N}_{3} \mathrm{OClBr} \mathrm{C}, 70.32$; H,9.52; N, 11.12.Found: C, 69.32; H, 8.32; N, 10.32. Yield: 74\% , M.P: $174-176^{0} \mathrm{C}$.

Preparation of 4-(bromomethyl)-N-(4-(4-chlorophenyl)-6-methylpyrimidin-2-yl)benzamide (6g).

IR (KBr) $\left(\mathbf{c m}^{-1}\right) v_{\max }: 3410,3135,2986,2583,2112,1765,1435,1090,968,695 .{ }^{1} \mathbf{H}$ NMR (DMSOd6, $300 \mathrm{MHz}) \boldsymbol{\delta}: 1.18\left(\mathrm{~s}, 3 \mathrm{H},-\mathrm{CH}_{3}\right), 2.50\left(\mathrm{~s}, 2 \mathrm{H}, \mathrm{CH}_{2}\right), 4.02(\mathrm{~s}, 1 \mathrm{H}, \mathrm{NH}), 6.28(\mathrm{~s}, J=7.8 \mathrm{~Hz}$, 1H,Ar-H),6.8-7.83(m,10H,Ar-H). ${ }^{13} \mathbf{C} \quad$ NMR (DMSO-d6,75 MHz) $\boldsymbol{\delta}: 14.6,25.1,114.9,121.6$, 125.8,126.6,127.8,129.2,134.4,137.8,141.4,143.6,144.8,152.8,156.9,164.8,168.1,170.1.Mass(C $\left.\mathrm{HCl}_{3}\right) \lambda_{\max / \mathrm{nm}\left(\mathrm{cm}^{-1}\right): \mathrm{m} / z} 413.5\left(M^{+}\right), 415.5(M+2)$.Anal. Calcd. for $\mathrm{C}_{19} \mathrm{H}_{15} \mathrm{~N}_{3} \mathrm{OClBr} \mathrm{C}, 71.02 ; \mathrm{H}$, 9.45; N, 10.20. Found: C,69.43; H,8.32; N, 10.01. Yield: 72\%, M.P: $172-174^{0} \mathrm{C}$.

Preparation of 4-(bromomethyl)-N-(4-methyl-6-(4-nitrophenyl)pyrimidin-2-yl)benzamide (6h). IR (KBr) (cm-1) $v_{\max }$ : 3425,3145,2915,2510,2123,1765,1430,1230,922,667. ${ }^{\mathbf{H}}$ NMR (DMSOd6, $300 \mathrm{MHz}) \boldsymbol{\delta}: 1.23\left(\mathrm{~s}, 3 \mathrm{H},-\mathrm{CH}_{3}\right), 2.50\left(\mathrm{~s}, 2 \mathrm{H}, \mathrm{CH}_{2}\right), 4.01(\mathrm{~s}, 1 \mathrm{H}, \mathrm{NH}), 6.28(\mathrm{~s}, J=7.8 \mathrm{~Hz}, 1 \mathrm{H}, \mathrm{Ar}-$ $\mathrm{H}), 6.8-7.8(\mathrm{~m}, 10 \mathrm{H}, \mathrm{Ar}-\mathrm{H}) .{ }^{13} \mathbf{C} \quad$ NMR $\quad$ (DMSO-d6,75 $\mathrm{MHz}$ ) $\quad \boldsymbol{\delta}: 12.6,23.1,110.9,112.8$, 121.8,122.2,126.8,128.5,130.3,132.6,134.4,136.7,137.8,141.4,144.5,150.8,156.9,164.8,167.9.Ma

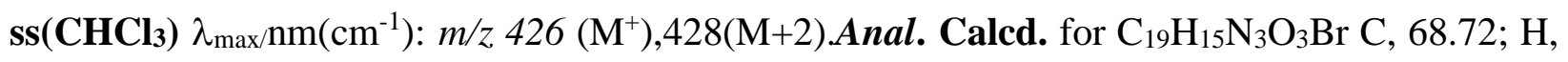
9.32; N, 10.11. Found: C, 67.72; H, 8.42; N, 10.01. Yield: 68\% , M.P: $162-164^{0} \mathrm{C}$.

Preparation of 4-(bromomethyl)-N-(4-methyl-6-p-tolylpyrimidin-2-yl)benzamide (6i).

$\operatorname{IR}(\mathbf{K B r})\left(\mathbf{c m}^{-\mathbf{1}}\right) v_{\max }: 3320,3165,2960,2530,2010,1756,1440,1190,942 .{ }^{\mathbf{1}} \mathbf{H}$ NMR (DMSO-d6, 300 $\mathrm{MHz}) \quad \boldsymbol{\delta}: 1.1\left(\mathrm{~s}, 3 \mathrm{H},-\mathrm{CH}_{3}\right), 1.86\left(\mathrm{~s}, 3 \mathrm{H}, \mathrm{CH}_{3}\right), 2.01\left(\mathrm{~s}, 2 \mathrm{H},-\mathrm{CH}_{2}\right), 3.98(\mathrm{~s}, 1 \mathrm{H}, \mathrm{NH}), 6.28(\mathrm{~s}, \quad J \quad=\quad 7.8$ $\mathrm{Hz}, 1 \mathrm{H}, \mathrm{Ar}-\mathrm{H}), 7.24-7.83(\mathrm{~m}, 10 \mathrm{H}, \mathrm{Ar}-\mathrm{H}) .{ }^{13} \mathrm{C} \quad$ NMR (DMSO-d6,75 MHz) $\boldsymbol{\delta}:$ 11.6,22.1,110.9, $112.8,121.8,122.2,126.8,128.5,129.3,131.6,134.4,136.7,137.8,141.4,143.5,150.8,156.9,163.8,168$ 


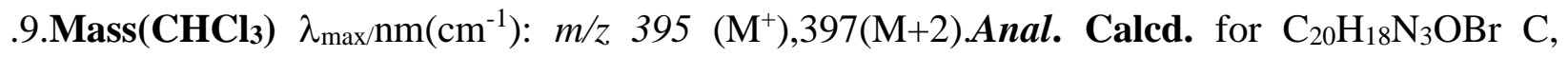
72.72; H, 10.32; N, 9.11. Found: C, 71.70; H, 9.42; N, 10.01. Yield: 62\%, M.P: $170-172^{0}$ C.

Preparation of 4-(bromomethyl)-N-(4-(4-fluorophenyl)-6-methylpyrimidin-2-yl)benzamide (6j).

IR (KBr) $\left(\mathbf{c m}^{-1}\right) v_{\max }: 3330,3075,2970,2640,2080,1708,1440,1190,942 .{ }^{1} \mathbf{H}$ NMR (DMSO-d6, $300 \mathrm{MHz}) \boldsymbol{\delta}: 1.20\left(\mathrm{t}, 3 \mathrm{H},-\mathrm{CH}_{3}\right), 2.35\left(\mathrm{~s}, 3 \mathrm{H}, \mathrm{CH}_{3}\right), 2.58\left(\mathrm{q}, 2 \mathrm{H},-\mathrm{CH}_{2}\right), 4.56\left(\mathrm{~s}, 2 \mathrm{H}, \mathrm{CH}_{2}\right), 6.28(\mathrm{~s}, J=7.8$ $\mathrm{Hz}, \quad$ 1H,Ar-H), 7.24-7.83(m,10H,Ar-H). ${ }^{13} \mathbf{C} \quad$ NMR (DMSO-d6,75 MHz) $\quad \boldsymbol{\delta}: \quad$ 12.6,20.1, 115.9,116.8,118.8,120.2,122.8,128.5,129.3,131.6,134.4,136.7,137.8,141.4,143.5,150.8,158.9,165 8, 169.9.Mass $\left(\mathbf{C H C l}_{3}\right) \lambda_{\max } / \mathrm{nm}\left(\mathrm{cm}^{-1}\right): \mathrm{m} / z 398\left(\mathrm{M}^{+}\right), 400(\mathrm{M}+2)$.Anal. Calcd. for $\mathrm{C}_{19} \mathrm{H}_{15} \mathrm{~N}_{3} \mathrm{OBrF}$ C,62.72; H, 11.32; N, 08.11. Found: C, 61.72; H, 10.42; N, 7.01. Yield: 64\%, M.P: 168-170 C.

Preparation of 4-(bromomethyl)-N-(4-(4-bromophenyl)-6-methylpyrimidin-2-yl) benzamide $(6 k)$.

IR (KBr) $\quad\left(\mathbf{c m}^{-1}\right) \quad v_{\max }: \quad 3330,3115,2910,2480,2110,1798,1340,1090,941,720,663 .{ }^{1} \mathbf{H} \quad$ NMR (DMSO-d6, $300 \mathrm{MHz}) \quad \boldsymbol{\delta}: 1.24\left(\mathrm{~s}, 3 \mathrm{H},-\mathrm{CH}_{3}\right), 2.32\left(\mathrm{~s}, 2 \mathrm{H}, \mathrm{CH}_{2}\right), 4.52(\mathrm{~s}, 1 \mathrm{H}, \mathrm{NH}), 6.58(\mathrm{~s}, \quad J=7.8$ $\mathrm{Hz}, 1 \mathrm{H}, \mathrm{Ar}-\mathrm{H}), 6.8-7.8(\mathrm{~m}, 10 \mathrm{H}, \mathrm{Ar}-\mathrm{H}) .{ }^{13} \mathbf{C} \quad$ NMR $\quad$ (DMSO-d6,75 MHz) $\mathbf{\delta}: \quad$ 12.6,20.1,115.9, 116.8,118.8,120.2,122.8,128.5,129.3,131.6,134.4,136.7,137.8,141.4,146.5,149.8,152.9,162.8,170

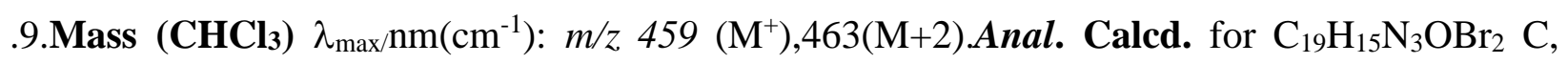
70.72; H, 9.32; N, 11.11. Found: C, 65.72; H, 7.42; N, 11.01.Yield: 60\% , M.P: $184-186^{0} \mathrm{C}$.

\section{ACKNOWLEDGEMENT}

The authors are grateful to the Head Department of Chemistry,, Osmania University, Hyderabad and Director, Indian Institute of Chemical Technology, Hyderabad, India, for providing NMR and Mass spectral data. Biological evaluation from the Department of Chemistry, Osmania University, Nizamabad, India, is greatly acknowledged.

\section{REFERENCES}

1. Mostafa, T. B. Synthesis and modification of some heterocyclic compounds with potential biological activity coupled on poly (maleic anhydride -methyl methacrylate). $J$. Am. Sci. 2010, 6, 512-524.

2. Singh, A. K.; Mishra, G.; Jyoti, K. Review on biological activities of 1,3,4-thiadiazole derivatives, J.Appl. Pharm. Sci. 2011, 1, 44-49.

3. Salimon, J.; Salih, N.; Hussien, H.; Yousif, E. Synthesis and characterization of new heterocyclic compounds derived from 2-aminopyridine. Eur. J. Sci. Res. 2009, 31, 256264. 
4. Xu, P. F.; Zhang, Z. H.; Hui, X. P.; Zhang, Z. Y.; Zheng, R. L. Synthesis of triazoles, oxadiazoles and condensed heterocyclic compounds containing cinchopheny and studies on biological activity of representative compounds. J. Chin. Chem. Soc. 2004, 51, 315319.

5. Miliani,L.F.; Nielsen, O.H.; Andersen, P.S.; Girardin,S.E. Chronic inflammation: importance of NOD2 and NALP3 in interleukin-1b generation. Clin. Exp. Immunol.,2006, 147, 227-35.

6. Vodovotz, Y.;Csete, M.;Bartels, J.;Chang, S.;An,G. Translational Systems Biology of Inflammation. PLoSComput. Biol., 2008, 4, e1000014.

7. Salminen, A.;Hyttinen, J.M.T.;Kaarniranta,K. AMP-activated protein kinase inhibits NF$\kappa \mathrm{B}$ signaling and inflammation: impact on healthspan and lifespan. J. Mol. Med.,2011, $89,667-76$.

8. Xu, X.;Steere, R.R.;Fedorchuk, C.A.; Pang, J.;Lee, J.Y.;Lim, J.H.;Xu, H.;Pan, Z.K.;Maggirwar, S.B.;Li, J.D. Activation of Epidermal Growth Factor Receptor is Required for NTHi-Induced NF-kB-Dependent Inflammation. PLoS ONE.,2011, 6, e28216.

9. Lawrence,T. The Nuclear Factor NF-kB Pathway in Inflammation. Cold Spring Harb.Perspect. Biol.,2009, 1:a001651.

10. Nagaraj.A;Ravi.G;Nageshwara Rao.G; SharathKumar Goud.S; Naseem: Synthesis of New Biologically Active Compounds Containing Linked Thiazolyl-Thaizolidinone Heterocycles. Organic Communications, 2012,5(4),160-170.

11. Ravi. G; Ravinder Nath. A; Nagaraj .A; Damodhar.S; Nageshwara Rao.G: Synthesis and antibacterial activity of 3-(5-methyl-1-phenyl-1 $H$-1,2,3-triazol-4-yl)-6-aryl-7H[1,2,4]traizolo[3,4,-b][1,3,4]thiadiazine, Der Pharma Chemica, 2014,6(4),223-232.

12. Y.S. Agasimundin, Ujjinimatada Ravi K, G S Harwalkar, and N V Kalyani, Indian Journal of Chemistry.Vol. 39B, pp 587-591, (2000)

13. Raga Basawaraj, Srikanth Patil, Ashok Patil, T.Vijaykumar, G. Parmeshwarappa An Indian Journal OCAIJ, 5(1), (2009), pp 68-72

14. Vijaykumar Tirlapur, Raga Basawaraj and Y.Rajendra Prasad Indian Journal of Heterocyclic Chem. Vol 20 , pp. 49-52 (2010)

15. Vijay Kumar Tirlapur, Y.Rajendra Prasad and Raga Basawaraj Indian Journal of Heterocyclic Chem. Vol. 20, pp 57-60, (2010) 
16. Eicher, T., Hauptmann, S. \& Speicher, A. (2003). The Chemistry of Heterocycles Structure, Reactions, Syntheses and Applications; Wiley-vch Gmbh \& Co., Germany, pp 63-64.

17. Khan, W., Alam, M.J., Rashid, M., \& Chowdhury, R. (2005). A new structural alternative in benzo[b]furans for antimicrobial activity. Bioorganic \& Medicinal Chemistry, 13, 4796-4805.

18. Navidpoor, L.; Amni, M.; Shafarwoodi, H.; Abdi, K. J.; Ghahremani, M. H.; Shafiee, A. Bioorg. Med. Chem. Lett. 2006, 15, 4483.

19. Gao, Y. L.; Zhao, G. L.; Liu, W.; Shao, H.; Wang, Y. L.; Xu, W. R.; Tangand, L. D.; Wang, J. W. Indian j. Chem. 2010, 49B, 1499.

20. Ashoke, S.; Ramendra, P.; Priti, T.; Arvind, S.; Maulik, P. R.; Vishnu, J. R. Bioorg. Med. Chem. Lett. 2005, 15, 2115.

21. Gundugola, A. S.; Chandra, K. L.; Perchellet, E. M.; Waters, A. M.; Rayat, S. Bioorg. Med. Chem. Lett. 2010, 20, 3920.

22. Shiny, G.; Shanmugapandiyan, G. Int. J. Pharm. Pharmaceut. Sci. 2012, 4, 2102.

23. Madhusudana Reddy, M. B.; Bhoje Gowd, M.; Afzal Pasha, M. J. Chem. Sci. 2011, 123, 75.

24. Cantillo, D.; Gutmann, B.; Kappe, C.O. J. Am. Chem. Soc. 2011, 133, 4465.

25. El Kaim, L.; Grimaud, L.; Patil, P. Org. Lett. 2011, 13, 1261. 